\title{
Role of Magnetic Resonance Imaging in the Evaluation of Breast Cancer Response to Neoadjuvant Chemotherapy
}

\author{
GIORGIA PASQUERO ${ }^{1}$, ALESSANDRA SURACE ${ }^{2}$, ANTONIO PONTI ${ }^{3}$, \\ MASSIMILIANO BORTOLINI ${ }^{4}$, DONATELLA TOTA ${ }^{5}$, MARIA PIERA MANO $^{3}$, \\ RICCARDO ARISIO $^{6}$, CHIARA BENEDETTO ${ }^{1}$ and MARIA GRAZIA BAÙ ${ }^{7}$ \\ ${ }^{1}$ Gynecology and Obstetrics 1, Department of Surgical Sciences, \\ City of Health and Science, University of Turin, Turin, Italy; \\ ${ }^{2}$ Gynecology and Obstetrics 2, Department of Surgical Sciences, \\ City of Health and Science, University of Turin, Turin, Italy; \\ ${ }^{3}$ AOU Città della Salute e della Scienza, CPO Piemonte and EUSOMA Data Centre, Turin, Italy; \\ ${ }^{4}$ Breast Unit Surgery, Martini Hospital, Turin, Italy; \\ ${ }^{5}$ Radiology, Department of Diagnostic Imaging and Radiotherapy, \\ City of Health and Science, University of Turin, Turin, Italy; \\ ${ }^{6}$ Pathology, Department of Laboratory Medicine, City of Health and Science, University of Turin, Turin, Italy; \\ ${ }^{7}$ Gynecology and Obstetrics 3, Department of Surgical Sciences, \\ City of Health and Science, University of Turin, Turin, Italy
}

\begin{abstract}
Background/Aim: The aim of the study was to evaluate whether residual tumor assessment by magnetic resonance imaging (MRI) after neoadjuvant chemotherapy (NACT) is fundamental for a successive surgical strategy. Patients and Methods: We collected 55 MRIs performed after NACT. Results: Pathological response rate was $20 \%$. MRI's sensitivity, specificity, PPV and NPV were 50\%, 88\%, 54\% and $86 \%$, respectively. We observed a high variability between the different subgroups, with high number of false positives in luminal A/B tumors. Triple negative and HER2+ tumors had almost the same specificity and sensitivity (81\% and 50\%). Nevertheless, in the HER2+ group, PPV was greater than that in the triple negative group $171 \%$ and $33 \%$ respectively) and the NPV of the triple negative group was greater than that of the HER2+ one $190 \%$ and $64 \%$, respectively). Statistical analysis showed a weak but significant correlation between MRI and pathological
\end{abstract}

This article is freely accessible online.

Correspondence to: Alessandra Surace, MD, Department of Gynecology, AOU Città della Salute e della Scienza, University of Turin, Corso Spezia 60, 10126 Turin, Italy. Tel: +39 3490941770, e-mail: alessandra.sur@gmail.com

Key Words: Magnetic resonance imaging, breast neoplasms, neoadjuvant chemotherapy, pathological complete response (pCR), imaging complete response (iCR), breast cancer subtypes. assessment of residual tumor dimension. Conclusion: The present study, confirms literature data about MRI accuracy in diagnosing HER2+ and triple negative tumors, but suggests caution in case of luminal tumors' evaluation.

Breast magnetic resonance imaging (MRI) is the most sensitive of the available imaging modalities to characterize breast cancer. The size of the tumor invasive component estimated by MRI closely correlates with that determined with pathological examination. NACT indications are increasing in the last years and the residual tumor assessment is fundamental for the successive surgical strategy (1). MRI can detect small residual cancer nests after neoadjuvant chemotherapy (NACT), but it may overestimate the extent of the residual tumor in some cases (2). However, MRI appears more useful than clinical examination, mammography and ultrasound in determining residual tumor (3-5). Furthermore, its use is recommended by most guidelines because, although it has similar accuracy with breast ultrasound, MRI evaluates better multifocal, multicenter and contralateral tumors (6).

Breast cancers are sub-classified according to immunophenotype in luminal A: estrogen receptors and/or progesterone receptors positive $(\mathrm{ER} / \mathrm{PgR}+), \mathrm{Ki} 67<20, \mathrm{HER} 2$ negative, luminal B HER2 negative (ER+ and/or PgR+, Ki67 $\geq 20$, HER2 negative), luminal B HER2 positive (ER+ and/or PgR+, any Ki67, HER2 positive), non-luminal HER2+ (ER-, PR-, HER2+), and triple-negative (ER-, PR-, HER2 negative). Luminal, HER2+ and triple negative tumors imaging are different from each other. Luminal A/B subtypes 


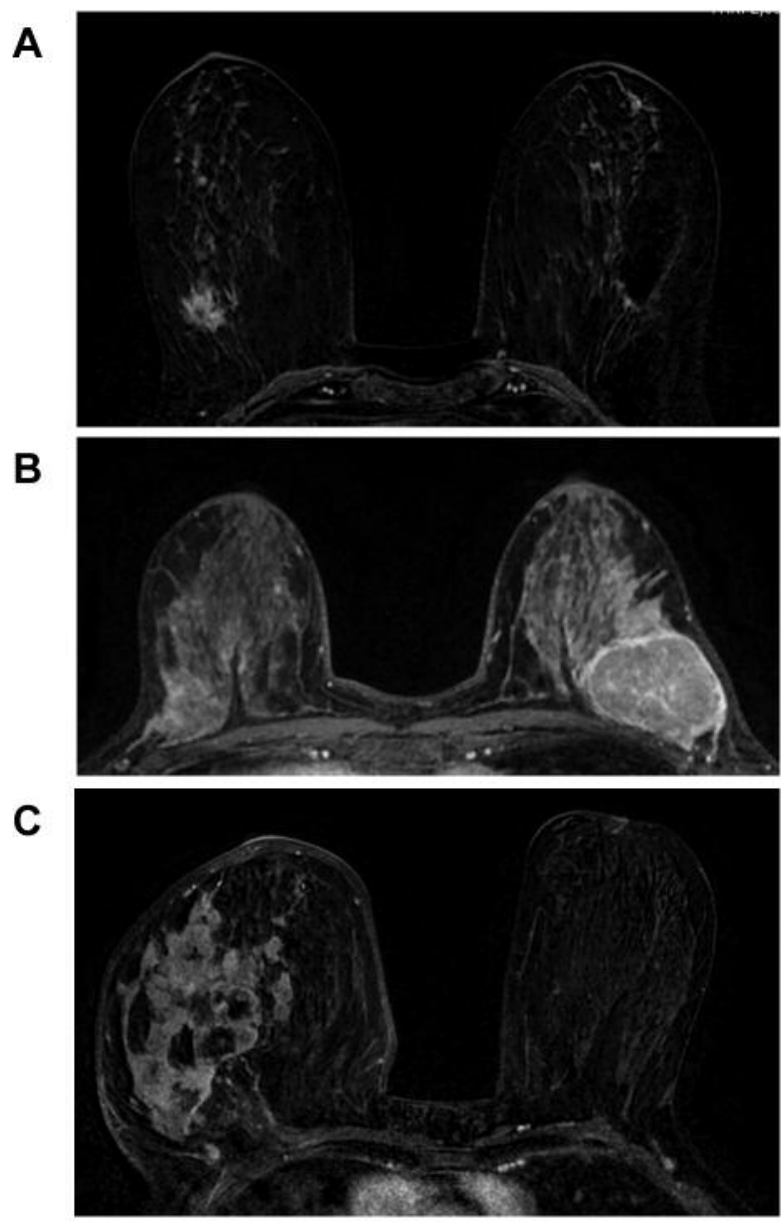

Figure 1. Breast cancer MRI appearances. A: Luminal A/B tumor: mass with irregular margins. B: HER2+ tumor: mass with regular margins. $C$ : Triple negative tumor: mass with intra-tumoral necrosis.

appear more frequently as a mass with irregular margins, while HER2+ tumors often have regular margins and the presence of intra-tumoral necrosis suggests a triple negative immunophenotype (Figure 1) (7). Stratifying the different tumor subtypes (luminal A/B, HER2+, triple negative), MRI's predictive role of $\mathrm{pCR}$ is higher for triple negative, HER2+ and "mass forming" morphology tumor (8-11). Association of MRI with diffusion weighted MRI, positron emission tomography - computed tomography (PET-CT) or breast ultrasound can give a more precise indication of $\mathrm{pCR}$, especially for HER2+ subtypes (12-13). Currently, surgical treatment after NACT is based on tumor response, requiring the removal of the residual tumor and not the whole initial tumor bed (14). It is necessary to avoid false negative and false positive imaging complete response (iCR) that could affect the surgical strategy and patient prognosis (15), and identify those cases in which MRI is actually predictive for



Figure 2. Sample selection.

Table I. Comparison between MRI and histological evaluation of complete or not complete response to NACT. iCR: Imaging complete response; $p C R$ : pathological complete response; TN: true negative; TP: true positive; FN: false negative; FP: false positive.

\begin{tabular}{lccc}
\hline & \multicolumn{3}{c}{ Histological reports } \\
\cline { 2 - 4 } & $\mathrm{pCR}$ & no pCR & Total \\
\hline MRI & & & \\
iCR & $6(\mathrm{TN})$ & $6(\mathrm{FN})$ & 12 \\
No iCR & $5(\mathrm{FP})$ & $38(\mathrm{TP})$ & 43 \\
Total & 11 & 44 & 55 \\
\hline
\end{tabular}

pCR. The main aim of our study is to estimate MRI accuracy in finding a complete response to NACT. MRI predictive values (positive and negative, PPV and NPV respectively), sensitivity and specificity are calculated comparing MRI reports of $\mathrm{iCR} /$ no iCR (radiological images of residual tumor) and post-surgical histological reports of $\mathrm{pCR} /$ no $\mathrm{pCR}$ (residual tumor on specimen). Besides that, we conducted the same analysis by dividing the whole sample in three subgroups (luminal A/B, HER2+, triple negative tumors). We assessed MRI accuracy in residual tumor dimensions definition.

\section{Patients and Methods}

We collected all MRIs performed between 2015 and 2017 in the Breast Unit of Sant' Anna Hospital in Turin, Italy. The inclusion criteria for this study were: female patients with biopsy-proven breast cancer; $\geq 18$ years old; not pregnant \& not breastfeeding; treated with NACT at the same hospital with MRI performed after completion of the treatment. 
Pasquero et al: Breast Magnetic Resonance Imaging After Neoadjuvant Chemotherapy

Table II. Sensitivity, specificity, positive predictive value (PPV) and negative predictive value (NPV) of the whole cohort and of each subgroup.

\begin{tabular}{lccrr}
\hline & $\begin{array}{c}\text { MRI (Triple negative+HER2 } \\
\text { LUMINAL A/B) }\end{array}$ & Triple Negative & LER2+ \\
\hline Sensitivity (\%) & 50 & 50 & 50 & 100 \\
Specificity (\%) & 88.4 & 81 & 81 & 0 \\
PPV (\%) & 54 & 33 & 71 & 64 \\
NPV (\%) & 86 & 90 & 65 \\
\hline
\end{tabular}

Table III. Comparison between MRI and pathological evaluation of complete or not complete response to NACT in luminal A/B tumors group. iCR: Imaging complete response; $p C R$ : pathological complete response.

\begin{tabular}{lccc}
\hline & \multicolumn{2}{c}{ Histological reports (luminal A/B tumors) } \\
\cline { 2 - 4 } & pCR & No pCR & Total \\
\hline MRI & & & \\
iCR & 0 & 0 & 0 \\
No iCR & 1 & 20 & 21 \\
Total & 1 & 20 & 21 \\
\hline
\end{tabular}

Table IV. Comparison between MRI and pathological evaluation of complete or not complete response to NACT in triple negative tumors group. iCR: Imaging complete response; $p C R$ : pathological complete response.

\begin{tabular}{llcc}
\hline & \multicolumn{3}{c}{ Histological reports (triple negative tumors) } \\
\cline { 2 - 4 } & pCR & No pCR & Total \\
\hline MRI & & & \\
iCR & 1 & 1 & 2 \\
No iCR & 2 & 9 & 11 \\
Total & 3 & 10 & 13 \\
\hline
\end{tabular}

Patients were excluded if they had undergone MRI or any treatment at another hospital and if there were contraindications for MRI or MRI contrast agents. Ten patients were excluded because they underwent surgery or chemotherapy at another hospital. Therefore, a total of 55 patients were included in this study. Patients median age was 56 years old (range $=32-80$ years). Patients cohort was composed of 21 luminal A/B tumors, 13 triple negative tumors, and 21 HER2 positive tumors. In luminal A/B group, 13 patients out of 21 were HER2 enriched and 8 were HER2 negative (Figure 2). We administered NACT according to this schedule: epirubicin $75-90 \mathrm{mg} / \mathrm{mq}$ and cyclophosphamide 600 $\mathrm{mg} / \mathrm{mq}$ for 4 cycles every 3 weeks followed by paclitaxel $80 \mathrm{mg} / \mathrm{mq}$ weekly for 12 weeks, adding trastuzumab in HER2 positive tumors. At the end of the treatment, MRI was performed with an Open MRI Hitachi 0.4 Tesla, MRI Philips Ingenia 1.5 Tesla or MRI Philips Achieva D-Stream 1.5 Tesla. Patients were in a prone position to
Table V. Comparison between MRI and pathological evaluation of complete or not complete response to NACT in HER2 + tumors group. iCR: Imaging complete response; $p C R$ : pathological complete response.

\begin{tabular}{llcc}
\hline & \multicolumn{3}{c}{ Histological reports (HER2+ tumors) } \\
\cline { 2 - 4 } & $\mathrm{pCR}$ & No pCR & Total \\
\hline MRI & & & \\
iCR & 5 & 5 & 10 \\
No iCR & 2 & 9 & 11 \\
Total & 7 & 14 & 21 \\
\hline
\end{tabular}

Table VI. Mean, trend, median of tumor dimension evaluated by MRI and post-operative histological report (HR).

\begin{tabular}{lcc}
\hline & MRI (mm) & HR (mm) \\
\hline Mean & 29.85 & 18.33 \\
Trend & 15.00 & 15.00 \\
Median & 22.00 & 15.00 \\
\hline
\end{tabular}

obtain images before and after administration of Gadobutrole (GadovistTM $1 \mathrm{mmol} / \mathrm{ml}) 0.1 \mathrm{mmol} / \mathrm{kg}(0.1 \mathrm{ml} / \mathrm{kg}$ ) at an injection rate of $2 \mathrm{ml} / \mathrm{s}$. Conservative or radical breast surgery was performed in 33 and 22 patients, respectively. We collected MRI reports of complete response (iCR) or not complete response (no iCR) to NACT and we compared them with post-operative histological analysis of $\mathrm{pCR}$ or persistence of residual tumor (no pCR). We conducted a statistical analysis first on the whole sample and then on each subgroup (luminal $\mathrm{A} / \mathrm{B}$, triple negative, HER2 tumors). We used a $2 \times 2$ contingency table (Table I) and calculated specificity, sensitivity, positive predictive value (PPV) and negative predictive value (NPV).

\section{Results}

We defined sensitivity as the proportion of patients with pCR that were correctly classified by MRI as complete responders (iCR). Specificity was defined as the proportion of patients with residual disease (no pCR) correctly classified by MRI 


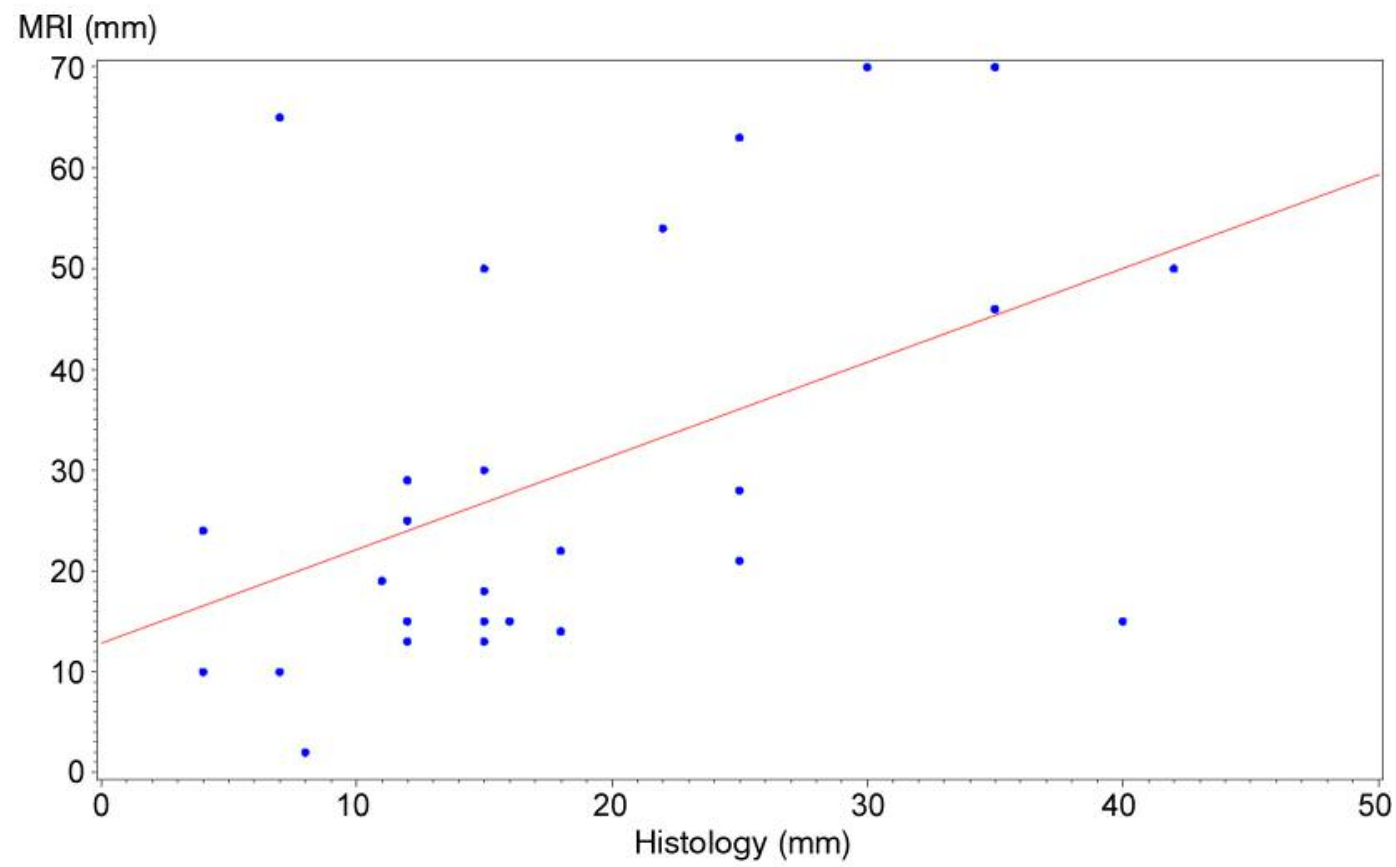

Figure 3. Correlation between residual tumor dimension assessment (MRI vs. pathology) (Pearson $r=0.48, p=0.01)$.

as such (no iCR). We tested the association between $\mathrm{iCR}$ and pCR $(\chi$-square $=8.63, p=0.0003)$. The pathological response rate to NACT was $20 \%$ (11 out of 55 cases). Among cases that responded completely (pCR), 6 out of 11 were correctly identified as iCR by MRI (TN), but 5 out of 11 were wrongly identified as not iCR by MRI (FP; 45\%). Among cases that responded partially to NACT (not pCR), 6 out of 44 were wrongly identified as iCR by MRI (FN; $13 \%)$, but 38 out of 44 were correctly identified as not iCR by MRI (TP). MRI's sensitivity, specificity PPV and NPV, were $50 \%$, $88 \%, 54 \%$ and $86 \%$, respectively. After subtype stratification, we observed a high variability in sensibility, specificity, PPV and NPV values between the different subgroups (Table II). MRI leads to non-specific and heterogeneous responses with a high number of false positives in luminal A/B tumors (Table III). Triple negative and HER2+ tumors (Tables IV and V) had almost the same specificity and sensitivity (81\% and 50\%). Nevertheless, PPV was greater in the HER2+ group compared to the triple negative group (71\% and $33 \%$, respectively) and the triple negatives' group NPV was greater than that of the HER2+ one (90\% and 64\%, respectively). Finally, in the subgroup of no pCR patients, we analyzed the correlation between MRI and pathologic assessment of residual tumor dimension, calculating the Pearson's r, p, mean, trend and median for both the complete patient series (Table VI) and the three different subgroups. Statistical analysis of the whole cohort showed a weak but significant correlation between MRI and pathological assessment of residual tumor dimension (Figure 3). Stratification in the three subgroups did not result in statistically significant differences (Figure 4), however, the correlation was very low $(0.23)$ in the group of luminal tumors (the largest group of our series).

\section{Discussion}

In our study, MRI after NACT had a sensitivity, specificity, PPV and NPV of $50 \%, 88.4 \%, 54 \%$ and $86 \%$, respectively. These data agree with the current literature, in particular with a systematic review published in 2013 (3). Our analysis revealed that MRI sensitivity and specificity values are very variable between the three histotypes. MRI has a very low specificity in predicting $\mathrm{pCR}$ in luminal A/B tumors, so, in this subgroup, surgery strategy cannot rely only on MRI data. Nevertheless, MRI specificity was higher (81\%) in HER2+ subgroup than in luminal A/B one. MRI sensitivity and specificity were $50 \%$ and $81 \%$, respectively, in triple negative group analysis. Our data agrees with literature: MRI predicts better pCR in triple negative and HER2+ tumors. However, our study deviates slightly from the other studies that have reported higher levels of sensitivity, specificity and PPV $(88.9 \%, 91.4 \%$ and $72.7 \%$ respectively) in triple negative analysis (8). Evaluating this data, subgroups dimension discrepancy (with lower percentage of triple negatives compared to the others subgroups) and the different numbers of pCR in luminal $\mathrm{A} / \mathrm{B}$, triple negative and HER2+ tumors $(5 \%$, $23 \%$ and $33 \%$, respectively) must be considered. 


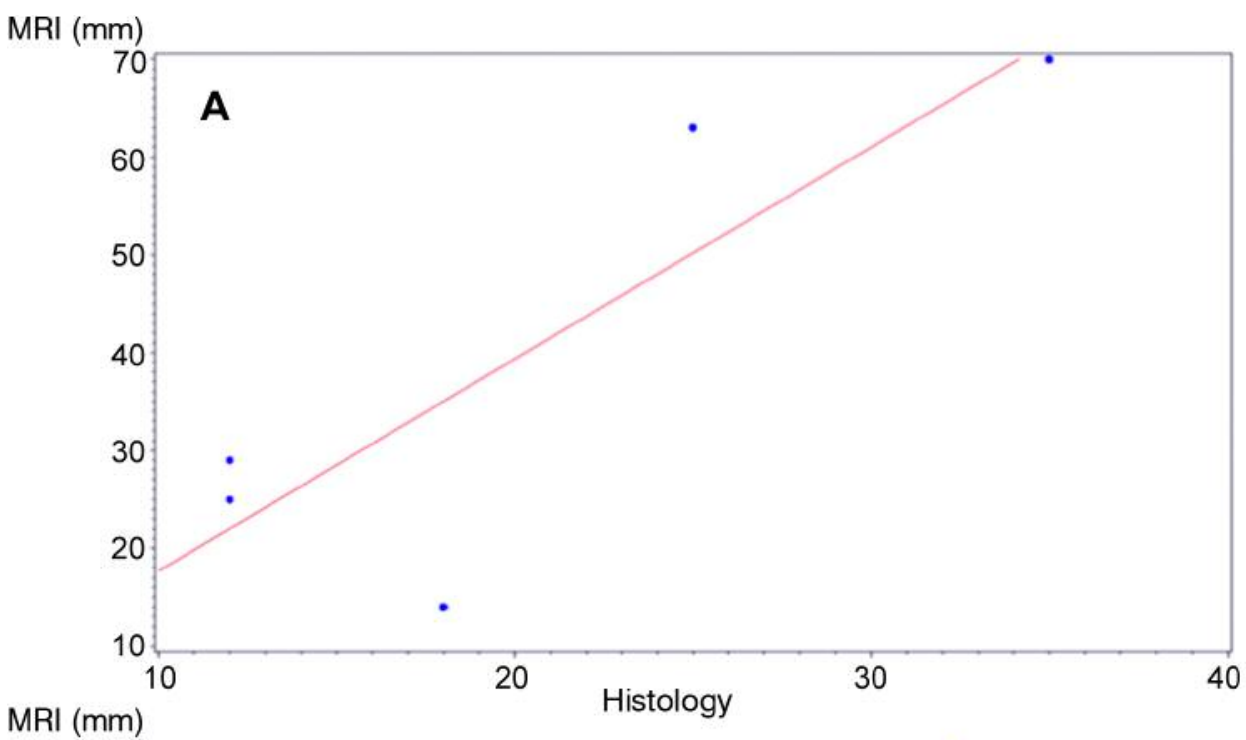

MRI (mm)



MRI (mm)

Histology

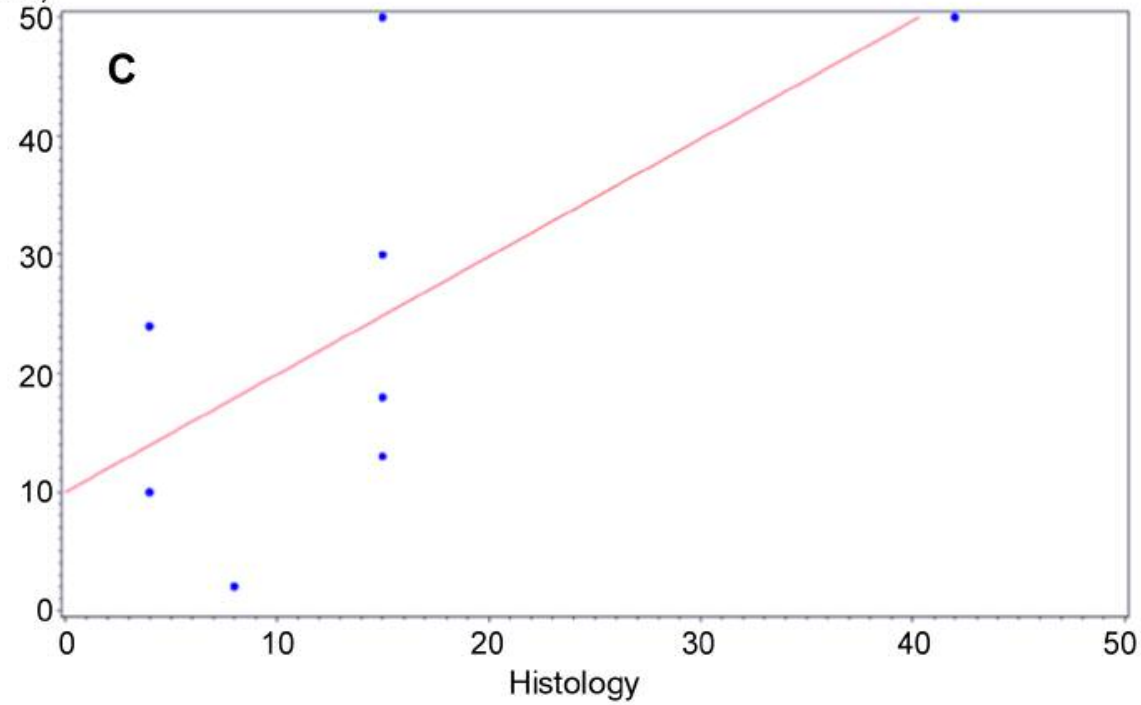

Figure 4. Correlation between residual tumor dimension assessment (MRI vs. pathology) in each tumor subgroup. A: Luminal A/B tumors (Pearson $r=0.23, p=0.42$ ). B: Triple negative tumors (Pearson $r=0.85, p=0.06)$. C: HER $2+$ tumors (Pearson $r=0.67, p=0.07$ ). 
Considering only patients who do not reach pCR after NACT and comparing MRI and pathologic assessment of residual tumor dimension, Pearson's $r$ was $0.48(p=0.01)$ which, although weakly, is statistically significant. Stratifying tumor subtypes, MRI accuracy did not seem reliable in luminal tumors response evaluation. Although this group is the largest one, the correlation was very low and not statistically significant. The correlation was higher and significant in triple negative and HER2+ tumor assessment (0.85 and 0.67, respectively). NACT indications were increasing and the successive surgical approach depended on residual tumor and not on initial tumor bed, so a reliable predictive method of pCR is strongly advisable. Our study, confirms literature data about MRI accuracy in HER2+ and triple negative tumors, but suggests caution in the case of luminal tumors' evaluation. Therefore, looking at the results obtained, MRI has an FP rate of $45 \%$ and an $\mathrm{FN}$ rate of $13 \%$, thus leading to over-treatment in $45 \%$ and under-treatment in $13 \%$ patients. However, since recent literature indications for surgery approach after NACT might range from surgery abstention in iCR patients (16) to the asportation of the entire area involved at the beginning of medical treatment in not iCR patients, further histological examination could be suggested in association with MRI, especially in luminal subtypes.

\section{Conflicts of Interest}

The Authors have no conflicts of interest to declare regarding this study.

\section{Authors' Contributions}

Pasquero Giorgia: Substantial contributions to acquisition of data; Surace Alessandra: Substantial contributions to acquisition of data; Ponti Antonio: Substantial contributions to analysis of data; Bortolini Massimiliano: Revising the article critically for important intellectual content. Tota Donatella: Substantial contributions to acquisition of data. Mano Maria Piera: Substantial contributions to conception and design of the study. Arisio Riccardo: Substantial contributions to analysis of data. Benedetto Chiara: Substantial contributions to organization of the conduct of the study; Baù Maria Grazia: Substantial contributions to conception and design of the study.

\section{Acknowledgements}

This research did not receive any specific grant from funding agencies in the public, commercial, or not-for-profit sectors.

\section{References}

1 Hahn A, Schlotter CM, Rossmanith WG, Ulmer HU, Staiger HJ and Villena C: Neoadjuvant chemotherapy for breast cancer with weekly nab-paclitaxel followed by epirubicin and cyclophosphamide results of a case series. In Vivo 28(2): 235-241, 2014. PMID: 24632979 .
2 Kim HJ, Im YH, Han BK, Choi N, Lee J, Kim JH, Choi YL, Ahn JS, Nam SJ, Park YS, Choe YH, Ko YH and Yang JH: Accuracy of MRI for estimating residual tumor size after neoadjuvant chemotherapy in locally advanced breast cancer: relation to response patterns in MRI. Acta Oncol 26: 996-1003, 2007. PMID: 17851879 . DOI: 10.1080/02841860701373587

3 Lobbes MBI, Smidt M, Tjan-Heijnen VCG, Van Goethem M, Schipper R, Beets-Tan RG and Wildberger JE: The role of magnetic resonance imaging in assessing residual disease and pathologic complete response in breast cancer patients receiving neoadjuvant chemotherapy: a systematic review. Insights Imaging 4: 163-175, 2013. PMID: 23359240. DOI: 10.1007/s13244-013-0219-y

4 Park S, Yoon JH, Sohn J, Park HS, Moon HJ, Kim MJ, Kim EK, Kim SI and Park BW: Magnetic resonance imaging after completation of neoadjuvant chemotherapy can accurately discriminate between no residual carcinoma and residual ductal carcinoma in situ in patients with triple-negative breast cancer. PLoS One 11(2): e0149347, 2016. PMID: 26866475. DOI: 10.1371/journal.pone. 0149347

5 Lee SC, Grant E, Sheth P, Garcia AA, Bhushan Desai MB, Ji L, Groshen S, Hwang D, Yamashita M and Hovanessian-Larsen L: Accuracy of contrast-enhances ultrasound compared with magnetic resonance imaging in assessing the tumor response after neoadjuvant chemotherapy for breast cancer. J Ultrasound Med, 2017. DOI: 10.7863/ultra.16.05060

6 Vriens BE, de Vries B, Lobbes MB, van Gastel SM, van den Berkmortel FW, Smilde TJ, van Warmerdam LJ, de Boer M, van Spronsen DJ, Smidt ML, Peer PG, Aarts MJ and Tjan-Heijnen VC: Ultrasound is at least as good as magnetic resonance imaging in predicting tumor size post- neoadjuvant chemotherapy in breast cancer. Eur J Cancer 52: 67-76, 2015. PMID: 26650831. DOI: 10.1016/j.ejca.2015.10.010

7 Kawashima H, Inokuchi M, Furukawa H, Ikeda $\mathrm{H}$ and Kiturama S: Magnetic resonance imaging features of breast cancer according to intrinsic subtypes: correlations with neoadjuvant chemotherapy effects. SpringerPlus 3: 240, 2014. PMID: 24892001. DOI: 10.1186/2193-1801-3-240

8 Fukuda, Horii R, Gomi N, Miyagi Y, Takahashi S, Ito Y, Akiyama F, Ohno S and Iwase T: Accuracy of magnetic resonance imaging for predicting pathological complete response of breast cancer after neoadjuvant chemotherapy: association with breast cancer subtype. SpringerPlus 5: 152, 2016. PMID: 27026849. DOI: 10.1186/s40064-016-1800-x

9 Michishita S, Kim SJ, Shimazu K, Sota Y, Naoi Y, Maruyama N, Kagara N, Shimoda M, Shimomura A and Noguchi S: Prediction of pathological complete response to neoadjuvant chemotherapy by magnetic resonance imaging in breast cancer patients. Breast 24: 159-165, 2015. PMID: 25805427. DOI: 10.1016/j.breast.2015.01.001

10 Price ER, Wong J, Mukhtar R, Hylton N and Esserman LJ: How to use magnetic resonance imaging following neoadjuvant chemotherapy in locally advanced breast cancer. World J Clin Cases 3(7): 607-613, 2015. PMID: 26244152. DOI: 10.12998/ wjcc.v3.i7.607

11 Ballesio L, Gigli S, Di Pastena F, Giraldi G, Manganaro L, Anastasi $\mathrm{E}$ and Catalano C: Magnetic resonance imaging tumor regression shrinkage patterns after neoadjuvant chemotherapy in patients with locally advanced breast cancer: correlation with tumor biological subtypes and pathological response after therapy. Tumor Biol 39(3), 2017. PMID: 28347225. DOI:i10.1177/1010428317694540 
$12 \mathrm{Gu}$ Y, Pan SM, Ren J, Yang ZX and Jiang GQ: Role of magnetic resonance imaging in detection of pathologic complete remission in breast cancer patients treated with neoadjuvant chemotherapy: a meta-analysis. Clin Breast Cancer 17(4): 245-255, 2017. PMID: 28209330. DOI: 10.1016/j.clbc.2016.12.010

13 Hayashi N. Tsunoda H, Namura M, Ochi T, Suzuki K, Yamauchi $\mathrm{H}$ and Namakura S: Magnetic resonance imaging combined with second-look ultrasonography in predicting pathologic complete response after neoadjuvant chemotherapy in primary breast cancer patients. Clin Breast Cancer 19(1): 71-77, 2018. PMID: 30206035. DOI: 10.1016/j.clbc.2018.08.004

14 Curigliano G, Burstein HJ, Winer EP, Gnant M, Dubsky P, Loibl S, Colleoni M, Regan MM, Piccart-Gebhart M, Senn HJ and Thurlimann B; Panel Members of the St. Gallen International Expert Consensus on the Primary Therapy of Early Breast Cancer 2017: De-escalating and escalating treatments for early-stage breast cancer: the St. Gallen International Expert Consensus Conference on the Primary Therapy of Early Breast Cancer 2017. Ann Oncol 28: 1700-1712, 2017. PMID: 28838210. DOI: 10.1093/annonc/mdx308
15 Dogan BE, Yuan Q, Bassett R, Guvenc I, Jackson EF, Cristofanilli $\mathrm{M}$ and Whitman GJ: Comparing the performances of magnetic resonance imaging size $v s$ pharmacokinetic parameters to predict response to neoadjuvant chemotherapy and survival in patients with breast cancer. Curr Probl Diagn Radiol 48(3): 235-224, 2018. PMID: 29685400. DOI: 10.1067/j.cpradiol.2018.03.003

16 Van der Noordaa MEM, Van Duijnhoven FH, Loo CE, van Werkhoven E, van de Vijver KK, Wiersma T, Winter-Warnars HAO, Sonke GS and Vrancken Peeters MTFD: Identifying pathological complete response of breast after neoadjuvant systemic with ultrasound guided biopsy to eventually omit surgery: Study design and feasibility of the MICRA trial (Minimally Invasive Complete Response Assessment). Breast 40: 76-81, 2018. PMID: 29698928. DOI: 10.1016/j.breast.2018.04.015

Received November 26, 2019

Revised December 12, 2019

Accepted December 18, 2019 